\title{
Response characteristics of array lateral logs and their primary inversion in reservoirs with fracture-induced anisotropy
}

\author{
Deng Shaogui ${ }^{*}$, Fan Yiren${ }^{1}$, Li Zhiqiang ${ }^{2}$ and Sun Qingtao ${ }^{1}$ \\ ${ }^{1}$ School of Geo-resources \& Information, China University of Petroleum, Shandong 266555, China
}

${ }^{2}$ China Research Institute of Radio Wave Propagation, Henan 453003, China

(C) China University of Petroleum (Beijing) and Springer-Verlag Berlin Heidelberg 2011

\begin{abstract}
In order to identify fractured reservoirs and determine their fracture parameters with a high definition array laterolog, we built a fracture-induced anisotropic formation model with a parallel fracture group. The three-dimensional finite element method is used to simulate the responses of the array laterolog, and then the primary inversion method is utilized. Numerical simulation shows that when the fracture spacing is small, the array laterolog response of the fracture group is the same as that of a formation with macroscopic electrical anisotropy. The apparent resistivity of the array laterolog is approximately inversely proportional to fracture porosity. The anisotropy depends on the fracture porosity in the fractured formation, which accordingly results in response variation of the array laterolog. The higher the fracture dip, the larger the apparent resistivity. When the fracture dip is low the difference between the deep and shallow apparent resistivities is small, and when the dip is high the difference turns out to be positive. The fracture parameters were inverted using the Marquardt non-linear least squares method. The results, both fracture porosity and dip show a good match with parameters in the actual formation model. This will promote the application of the array laterolog in evaluating fractured reservoirs.
\end{abstract}

Key words: Fracturing, anisotropic reservoir, fracture dip, fracture porosity, array laterolog

\section{Introduction}

Fractures not only create important storage space, but also provide good seepage channels for hydrocarbon migration. The degree of fracturing in fractured reservoirs has an important impact on reservoir productivity (Luo et al, 2001). Generally speaking, the resistivity values parallel to the fractures and normal to them are different, and the normal resistivity is commonly higher (Philippe and Roger, 1990; Zhao et al, 1994; Keller and Frischknecht, 1996; Klein and Martin, 1997; Bang et al, 2000), which results in electrical anisotropy in fractured reservoirs. Due to in-situ stress, fracturing commonly shows in the form of fracture groups, especially in fracture-developed zones, where the formation presents macroscopic electrical anisotropy. Electrical formation anisotropy leads to complex electrical logging response characteristics (Wang et al, 1998; Yang, 2003; Deng et al, 2006), so the evaluation of the reservoirs is difficult. In conventional logging, a dual laterolog can reflect the degree of fracturing effectively, because of its great electricity focusing ability. Currently, there is a trend of using a dual

*Corresponding author. email: dengshg@upc.edu.cn Received December 11, 2009 laterolog to determine the fracture porosity of formations (Sibbit and Faivre, 1985; Li et al, 1996). Compared with the dual laterolog, the novel array laterolog has higher resolution, provides more reservoir information and shows a good application potential (Chen et al, 1998; Smits et al, 1998; Liu and Zhang, 2005; Wu et al, 2008). In this paper, we simulated and analyzed the response characteristics of a novel array laterolog in reservoirs with fracture-induced electrical anisotropy by using a 3-D numerical method. Sensitive information on fractured reservoirs was obtained from the array laterolog and the response rules and characteristics are expected to have useful applications in the evaluation of fractured reservoirs.

\section{Calculation principles of the response of an array laterolog in a fracture-induced anisotropic medium}

\subsection{Plane model of fracturing}

Due to in-situ stress, fractures are commonly distributed in the form of a fracture group. This makes the formation resistivity anisotropic. A plane model of fracturing is established shown in Fig. 1, where fractures are evenly 


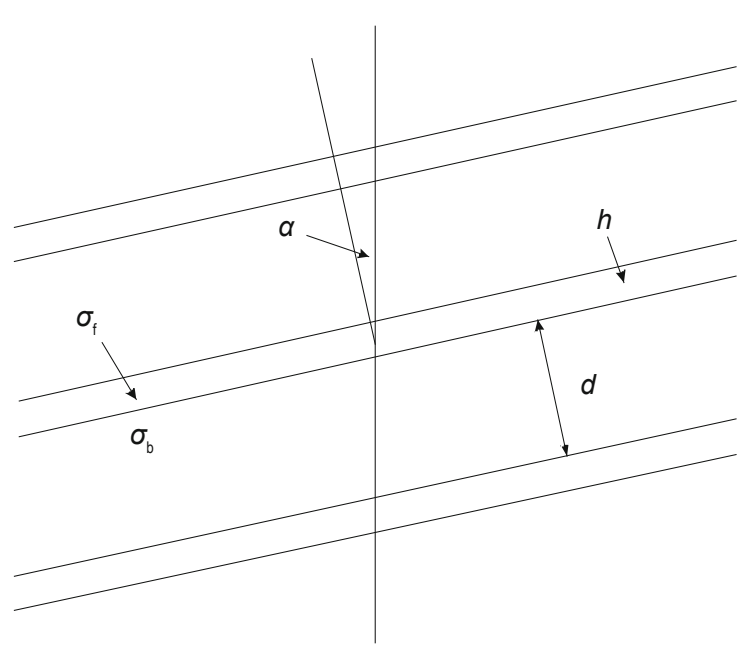

Fig. 1 Plane model of fractures

distributed in the whole region, $\sigma_{\mathrm{b}}$ and $\sigma_{\mathrm{f}}$ represent bulk conductivity of rock and fluid conductivity in fracture respectively, $h$ and $d$ represent fracture opening and spacing respectively, and $\alpha$ is fracture dip. When the fracture spacing is small enough, the conductivity of fractured formation shows macroscopic anisotropy (Li et al, 1996).

$\sigma_{\mathrm{h}}$ and $\sigma_{\mathrm{v}}$ represent the conductivity parallel and normal to the fracture respectively. When the fracture resistivity and bulk resistivity of rock are in parallel,

$$
\sigma_{\mathrm{h}}=\left(1-\phi_{\mathrm{f}}\right) \sigma_{\mathrm{b}}+\phi_{\mathrm{f}} \sigma_{\mathrm{f}}
$$

where, ${ }_{\mathrm{f}}$ is the fracture porosity, satisfying $\phi_{\mathrm{f}}=h /(h+d)$. When the fracture resistivity and bulk resistivity of rock are in series,

$$
1 / \sigma_{\mathrm{v}}=\left(1-\phi_{\mathrm{f}}\right) / \sigma_{\mathrm{b}}+\phi_{\mathrm{f}} / \sigma_{\mathrm{f}}
$$

Then, it can be obtained:

$$
\sigma_{\mathrm{v}}=\sigma_{\mathrm{b}} /\left[1+\phi_{\mathrm{f}}\left(\sigma_{\mathrm{b}} / \sigma_{\mathrm{f}}-1\right)\right]
$$

The conductivity of the fractured formation shows great anisotropy, and the anisotropy index $\gamma$ can be represented as follows:

$$
\gamma=\sqrt{\sigma_{\mathrm{h}} / \sigma_{\mathrm{v}}}
$$

The electrical anisotropy of fractured formation is mainly controlled by fracture porosity and fluid conductivity.

\subsection{Conductivity tensor of a fracture-induced anisotropic medium}

For an anisotropic medium, the differential form of Ohm's Law can be represented in the form of matrix and vector:

$$
j=\sigma E
$$

where $\boldsymbol{j}$ is the electric current density, $\boldsymbol{E}$ is the electric field intensity, and $\sigma$ is the medium conductivity tensor. In Cartesian coordinates, the equation is:

$$
\left[\begin{array}{c}
j_{x} \\
j_{y} \\
j_{z}
\end{array}\right]=\left[\begin{array}{ccc}
\sigma_{x x} & \sigma_{x y} & \sigma_{x z} \\
\sigma_{y x} & \sigma_{y y} & \sigma_{y z} \\
\sigma_{z x} & \sigma_{z y} & \sigma_{z z}
\end{array}\right]\left[\begin{array}{l}
E_{x} \\
E_{y} \\
E_{z}
\end{array}\right]
$$

By adopting a new coordinate system, the $\mathrm{X}^{\prime} \mathrm{Y}^{\prime}$ plane is made parallel to the fractures, and $Z^{\prime}$-axis is made normal to the fractures. The conductivity tensor $\sigma$ is simplified in the $\mathrm{X}^{\prime} \mathrm{Y}^{\prime} \mathrm{Z}^{\prime}$ coordinate system:

$$
\boldsymbol{\sigma}=\left[\begin{array}{ccc}
\sigma_{\mathrm{h}} & 0 & 0 \\
0 & \sigma_{\mathrm{h}} & 0 \\
0 & 0 & \sigma_{\mathrm{v}}
\end{array}\right]
$$

For a formation with dipping fractures, the position of the well axis differs relatively from that of the fracture normal line. The XYZ coordinate system is adopted, which is equivalent to rotating the $\mathrm{X}^{\prime} \mathrm{Y}^{\prime} \mathrm{Z}^{\prime}$ coordinate system by a specific degree (Fig. 2). Any vector $\boldsymbol{r}^{\prime}$ in the $\mathrm{X}^{\prime} \mathrm{Y}^{\prime} \mathrm{Z}^{\prime}$ coordinate system is changed to $\boldsymbol{r}$ in the $\mathrm{XYZ}$ coordinate system by rotation. The relationship between $\boldsymbol{r}^{\prime}$ and $\boldsymbol{r}$ can be represented by $\boldsymbol{R}: \boldsymbol{R} \boldsymbol{r}^{\prime}=\boldsymbol{r}$, and $\boldsymbol{R}$ satisfies:

$$
\boldsymbol{R}=\left[\begin{array}{ccc}
\cos \alpha \cos \beta & \cos \alpha \sin \beta & -\sin \alpha \\
-\sin \alpha & \cos \beta & 0 \\
\sin \alpha \cos \beta & \sin \alpha \sin \beta & \cos \alpha
\end{array}\right]
$$

where $\alpha$ is fracture dip, $\beta$ is fracture dip azimuth. Assuming $\beta=0$, then the conductivity tensor after rotation is:

$$
\boldsymbol{\sigma}=\left[\begin{array}{ccc}
\cos ^{2} \alpha \sigma_{\mathrm{h}}+\sin ^{2} \alpha \sigma_{\mathrm{v}} & 0 & \sin \alpha \cos \alpha\left(\sigma_{\mathrm{h}}-\sigma_{\mathrm{v}}\right) \\
0 & \sigma_{\mathrm{h}} & 0 \\
\sin \alpha \cos \alpha\left(\sigma_{\mathrm{h}}-\sigma_{\mathrm{v}}\right) & 0 & \sin ^{2} \alpha \sigma_{\mathrm{h}}+\cos ^{2} \alpha \sigma_{\mathrm{v}}
\end{array}\right]
$$

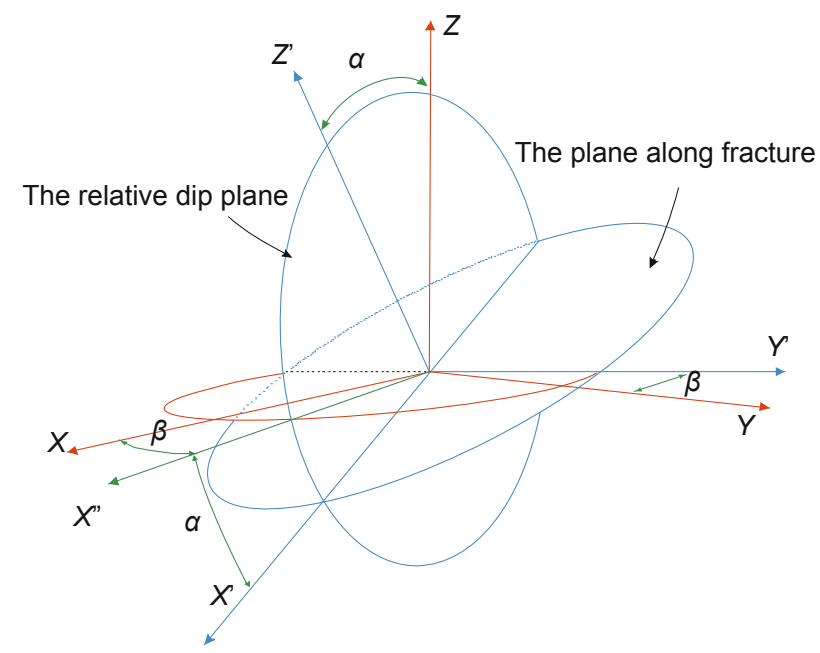

Fig. 2 Coordinate system of fore-and-aft rotation

2.3 Numerical solution of the response of array laterolog in a formation with fracture-induced anisotropy

An array laterolog is composed of one main electrode $\left(A_{0}\right)$, 


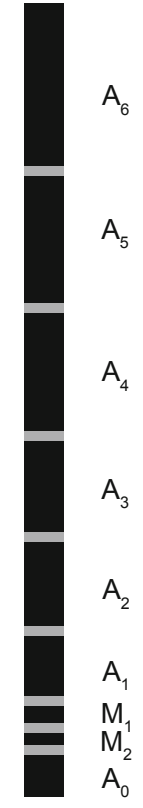

Fig. 3 Electrode arrangement of an array laterolog

six pairs of shielding electrodes $\left(\mathrm{A}_{1}, \mathrm{~A}_{2}, \ldots, \mathrm{A}_{6}\right)$, and two pairs of monitor electrodes $\left(\mathrm{M}_{1}, \mathrm{M}_{2}\right)$. Electrodes are symmetrically arranged with respect to the main electrode, and Fig. 3 shows the electrode arrangement above the main electrode. Six resistivities of array laterolog (RLA) with different investigation depths are provided. When $\mathrm{A}_{0}$ emits current and other shielding electrodes are used as circuit electrodes, $\mathrm{RLA}_{0}$ is obtained, which is used to determine mud resistivity in the borehole and is not discussed in this paper. By changing one pair of shielding electrodes into current emission electrodes in turn from both sides of $\mathrm{A}_{0}$ with other electrodes still as circuit electrodes, $\mathrm{RLA}_{1}, \mathrm{RLA}_{2}, \mathrm{RLA}_{3}, \mathrm{RLA}_{4}$ and $\mathrm{RLA}_{5}$ are formed in turn. Thereby six responses with different investigation depths are gained, which show good vertical resolution.

The continuous and smooth potential function $U$ in the response of array laterolog satisfies:

$$
\nabla \cdot(\sigma \nabla U)=0
$$

On the surface of constant voltage electrodes and constant current electrodes, $U$ satisfies the first boundary condition. On constant current electrodes, $U$ is an unknown constant. On the surface of constant current electrodes $E_{\mathrm{A}}, U$ satisfies the second boundary condition, $\int_{E_{\mathrm{A}}} \sigma_{\mathrm{m}} \frac{\partial U}{\partial n} \mathrm{~d} s=I$, and on the insulation boundary plane, $\frac{\partial U}{\partial n}=0$, where $I$ is current from supply electrode $E_{\mathrm{A}}$, and $\sigma_{\mathrm{m}}$ is mud conductivity. In order to solve Eq. (9), the partial differential equation is changed to calculate the extreme values of the functional:

$$
\Phi=\frac{1}{2} \iiint_{V} \boldsymbol{J} \cdot \boldsymbol{E} \mathrm{d} x \mathrm{~d} y \mathrm{~d} z-\sum_{E_{\mathrm{A}}} I_{E_{\mathrm{A}}} U_{E_{\mathrm{A}}}
$$

The physical meaning of the functional is power consumption in the calculation region minus the power provided by electrodes (Zhang, 1984). In Eq. (10), $V$ is the solution region, the remaining part of 3-D space outside the electrode system, $U_{E_{\mathrm{A}}}$ and $I_{E_{\mathrm{A}}}$ are respectively the electrode potential and supply current of the array laterolog. Then Eq. (10) is transformed to:

$$
\Phi=\frac{1}{2} \iiint_{V} \sum_{i, j=1}^{3}\left[\sigma_{i j} \frac{\partial U}{\partial \xi_{i}} \frac{\partial U}{\partial \xi_{j}}\right] \mathrm{d} x \mathrm{~d} y \mathrm{~d} z-\sum_{E_{\mathrm{A}}} I_{E_{\mathrm{A}}} U_{E_{\mathrm{A}}}
$$

where, $\sigma_{i j}$ is the $(i, j)$ element determined by conductivity tensor, $\xi_{1}=x, \xi_{2}=y$, and $\xi_{3}=z$. While computing, the solution region is divided into 274,176 tetrahedral elements. Improved frontal solution is adopted, making the computation speed very fast.

\section{Response characteristics of an array laterolog in a fracture-induced macro- anisotropic medium}

\subsection{The relationship between the array laterolog response of a parallel fracture group and that of a macro-anisotropic formation}

Fig. 4 shows the relationship between the array laterolog response of a parallel fracture group with the same spacing and different dips and that of a macro-anisotropic medium. The computation conditions are: bulk resistivity $R_{\mathrm{b}}$ is 10,000 $\Omega \cdot \mathrm{m}$, mud resistivity $R_{\mathrm{m}}$ is $0.1 \Omega \cdot \mathrm{m}$, borehole diameter $d_{\mathrm{h}}$ is $0.2 \mathrm{~m}$, fracture porosity $\mathrm{f}_{\mathrm{f}}$ is $0.05 \%$, and fluid resistivity in fractures $R_{\mathrm{f}}$ is $0.1 \Omega \cdot \mathrm{m}$. The discrete points in Fig. 4 represent the array laterolog response of the fracture group, and curves represent the response of the macro-anisotropic formation. The results suggest that when the fracture density is 10 per meter and the fracture dip is less than 30 degrees, the response of the macro-anisotropic formation matches well with that of the fracture group (Fig. 4(a)). When the fracture density is 20 per meter, for the whole dip range, they match well (Fig. 4(b)). Thus, when the spacing of the parallel fracture group is small enough, that is, fracturing is well developed, the responses of the array laterolog are the same, for both a macro-anisotropic formation and a microscopic fracture group.

\subsection{The effect of fracture porosity on the response of array laterolog}

Fig. 5 shows the relationship between the apparent resistivity and fracture porosity of an array laterolog in a fracture-induced anisotropic formation. This figure provides two cases with horizontal and vertical fractures, showing that when fracturing is well developed, the apparent resistivity is basically proportional to fracture porosity in the duallogarithm coordinate system except $\mathrm{RLA}_{1}$ which has a small investigation depth. When the fracture porosity is relatively small, the relationship between apparent resistivity and fracture porosity is non-linear. With fracture porosity increasing, the apparent resistivity decreases. This phenomena 


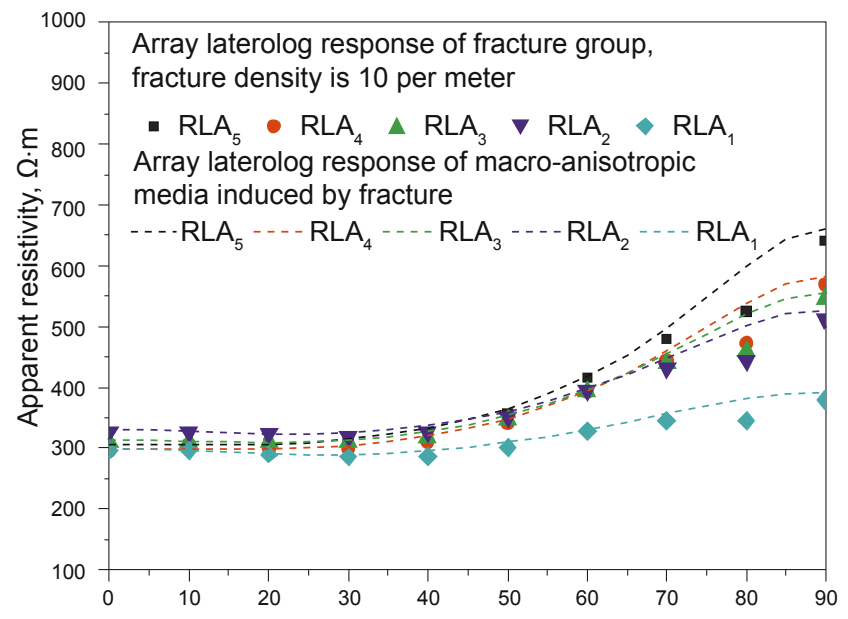

(a) Fracture dip, degree

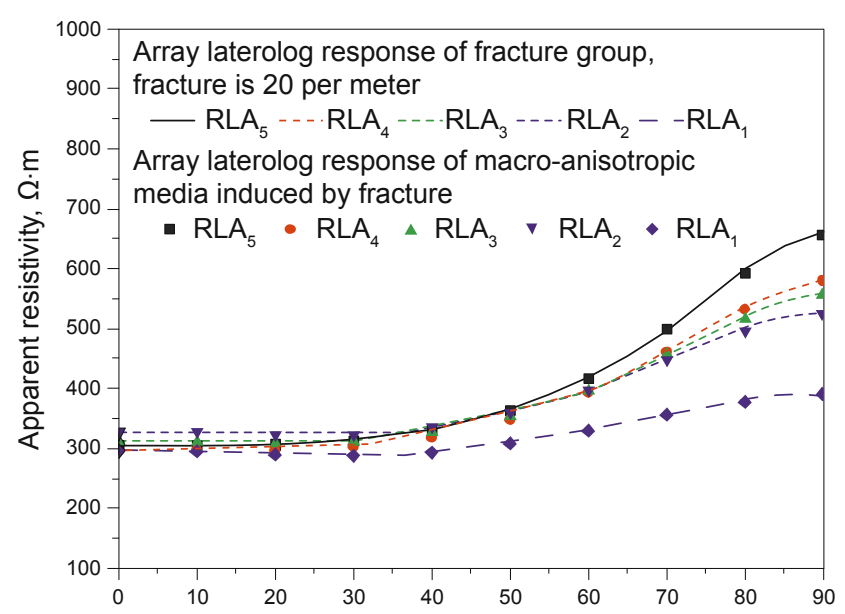

(b) Fracture dip, degree

Fig. 4 Array laterolog responses of a parallel fracture group and a macro-anisotropic formation

exists with any dip. When the fracture porosity is small, $\mathrm{RLA}_{1}$ is significantly lower than other responses due to the mud effect in the borehole rather than a greater sensitivity to fracturing.

In the porosity range simulated, deep resistivity is always higher than shallow resistivity in the formation with vertical fractures. In a formation with horizontal fracturing, they have little difference (except $\mathrm{RLA}_{1}$ ), and when fracturing is well developed, deep resistivity is smaller than shallow resistivity. The apparent resistivity of an array laterolog in a formation with vertical fractures is bigger than that in a formation with horizontal fractures under the same fracture porosity condition, which is due to fracture-induced anisotropy.

\subsection{The effect of fracture porosity on electrical anisotropy and the corresponding response of an array laterolog}

It is commonly believed that fractured formation is deeply invaded by mud, and there is little difference in fluid property in fractures. Therefore, fracture-induced anisotropy is mainly controlled by the degree of fracture development (Fig. 6). With fracture porosity increasing, the anisotropic

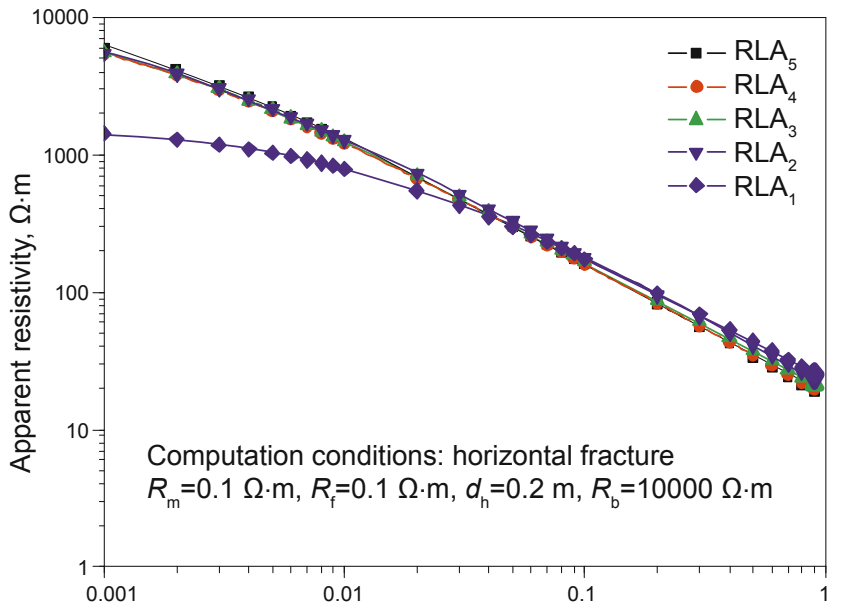

(a) Fracture porosity, \%

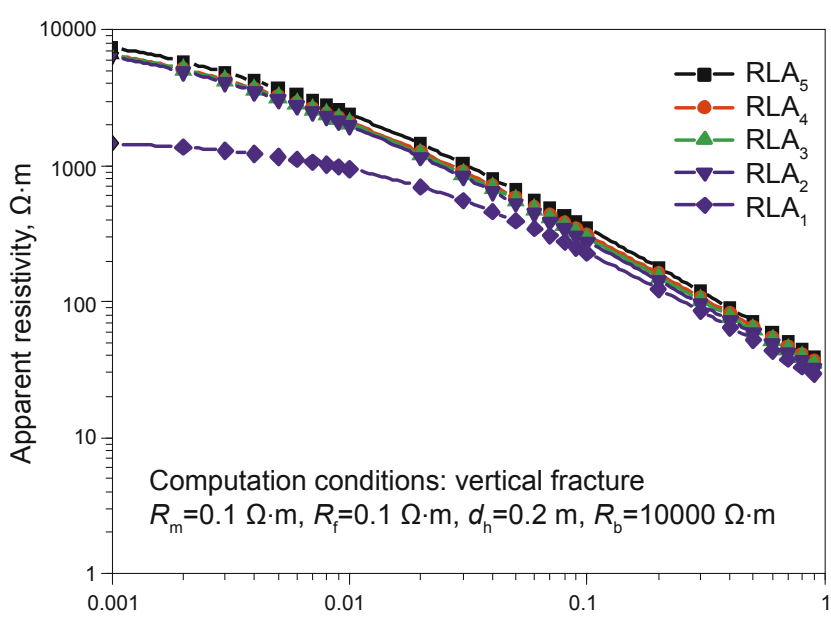

(b) Fracture porosity, \%

Fig. 5 Relationship between the array laterolog response and fracture porosity

index becomes bigger, thus resulting in changes of logging response (Fig. 7). When the fracture porosity is relatively small $(0.001 \%)$, the anisotropic index is about 1.4 , and the responses of the array laterolog under different dip conditions vary little. When the fracture porosity is large, because of the increasing anisotropic index, the response of array laterolog increases as the fracture dip increases. For example, when the fracture porosity is $0.01 \%$, the anisotropic index is about 3.3 ; when the fracture porosity is $0.05 \%$, the anisotropic index is about 7.1. For horizontal fracturing, the logging value mainly reflects the resistivity parallel to the fractures, and for vertical fracturing, the effect of formation resistivity along the fracture normal line on the logging value increases.

\section{Inversion of fracture parameters}

\subsection{Summary of inversion process}

Under certain bulk resistivity condition, fracture porosity $f$ (or the product of $f$ and fracture fluid conductivity ${ }_{\mathrm{f}} \cdot \sigma_{\mathrm{f}}$ ) and fracture dip $\alpha$ determine the response of an array laterolog, represented by the following mathematical function: 


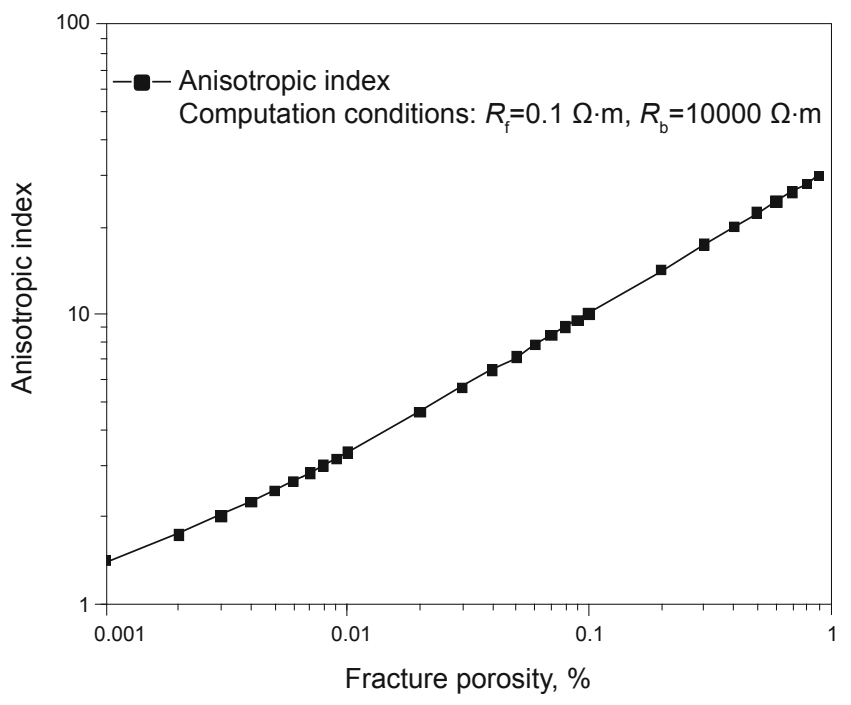

Fig. 6 Relationship between anisotropic index and fracture porosity

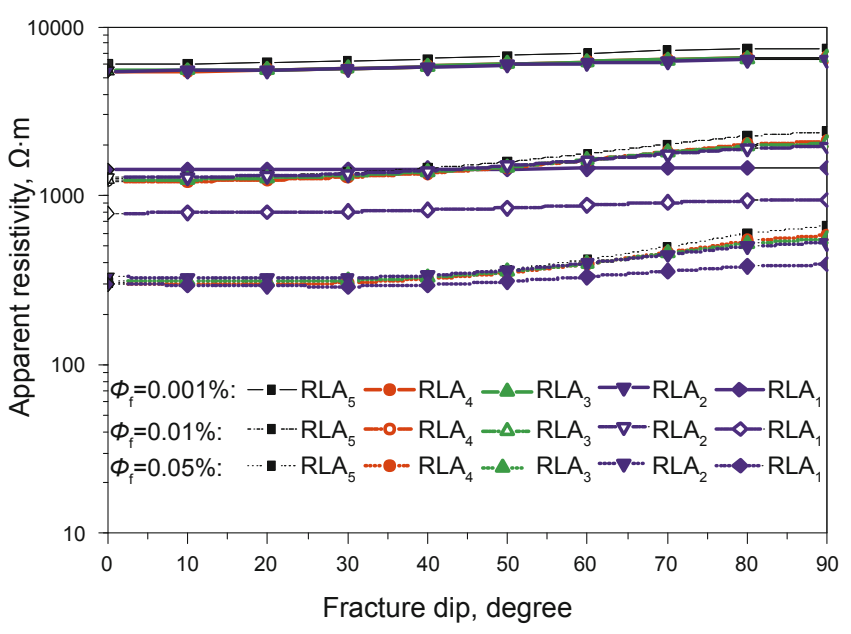

Fig. 7 Array laterolog response of a fractured anisotropic formation

$$
y_{i}=f_{i}(\boldsymbol{x}) \quad i=1, \ldots, n
$$

where, $\boldsymbol{x}=\left(x_{1}, x_{2}, x_{3}\right)$ is the formation parameter vector composed of fracture porosity, fracture dip and bulk resistivity, and $y_{i}$ is the array laterolog response of the $i^{\text {th }}$ point. Inversion of fracture parameter $\boldsymbol{x}$ using the array laterolog responses can be transformed into an optimized least square problem as follows:

$$
O=\sum_{i=1}^{n}\left(y_{i}-f_{i}(\boldsymbol{x})\right)^{2}
$$

Because a group of specific fracture porosity and dip can determine five array laterolog values with different investigation depths, $n(n \geq 2)$ responses are used for inversion. The least square problem in Eq. (13) is transformed into:

$$
\frac{\partial O}{\partial \boldsymbol{x}}=0
$$

Eq. (14) is non-linear and commonly can not be solved directly. Therefore, given the initial guessed value of model parameter $\boldsymbol{x}^{(0)}=\left(x_{1}{ }^{0}, x_{2}{ }^{0}, x_{3}{ }^{0}\right)$, the non-linear equation $y=f\left(x_{1}, x_{2}, x_{3}\right)$ is linearized by using Taylor's formula.

$$
f\left(x_{1}, x_{2}, x_{3}\right) \approx f_{i}\left(x_{1}^{0}, x_{2}^{0}, x_{3}^{0}\right)+\frac{\partial f_{i}}{\partial \boldsymbol{x}} \Delta \boldsymbol{x}
$$

where, $\Delta \boldsymbol{x}$ is equal to $\boldsymbol{x} \boldsymbol{x}^{(0)}$. Substitute Eq. (15) into Eq. (14), then:

$$
A \cdot \Delta x=b
$$

where, $\boldsymbol{A}$ satisfies:

$$
\boldsymbol{A}=\left(a_{i j}\right)=\left|\begin{array}{lll}
\frac{\partial f_{1}}{\partial x_{1}}, & \frac{\partial f_{1}}{\partial x_{2}}, & \frac{\partial f_{1}}{\partial x_{3}} \\
\frac{\partial f_{2}}{\partial x_{1}}, & \frac{\partial f_{2}}{\partial x_{2}}, & \frac{\partial f_{2}}{\partial x_{3}} \\
\cdots & \cdots & \cdots \\
\frac{\partial f_{n}}{\partial x_{1}}, & \frac{\partial f_{n}}{\partial x_{2}}, & \frac{\partial f_{n}}{\partial x_{3}}
\end{array}\right| \quad b_{i}=y_{i}-f_{i}
$$

In order to improve the convergence and stability of this algorithm, the Marquardt method is adopted:

$$
\begin{aligned}
& \left(A^{\prime} A+\lambda I\right) \Delta x=A^{\prime} b \\
& x=x+\Delta x
\end{aligned}
$$

Singular value decomposition is used to solve Eq. (17), and a damping factor $\lambda$ is induced to improve algorithm stability. During fracture inversion, $\lambda$ takes the value of 0.01 , and then is reduced continuously to improve convergence speed.

\subsection{Case study}

Under a specific bulk resistivity condition $\left(R_{\mathrm{b}}=1000\right.$ $\Omega \cdot \mathrm{m})$, the array laterolog responses with different fracture porosities and dips are determined. Meanwhile, white Gaussian noise with different signal-to-noise ratios (SNR) is added to simulate the measurement error while logging. Eqs. (16)-(18) are used to invert the responses of the array laterolog, in order to gain the fracture porosity and dip. In Fig. 8, the inversion results suggest that when the noise is very small, no matter the fracture porosity or dip, the inversion results coincide well with actual formation model. With increasing noise, the agreement decreases. When SNR reaches $30: 1$, the inverted fracture porosity is still reliable, but the difference between the inverted fracture dip and actual dip becomes relatively large, because the five logging responses with different investigation depths vary greatly due to the introduced noise. In other words, the difference is caused by different measuring accuracies, so the fracture parameters determined by the least square method deviate from the actual ones. 


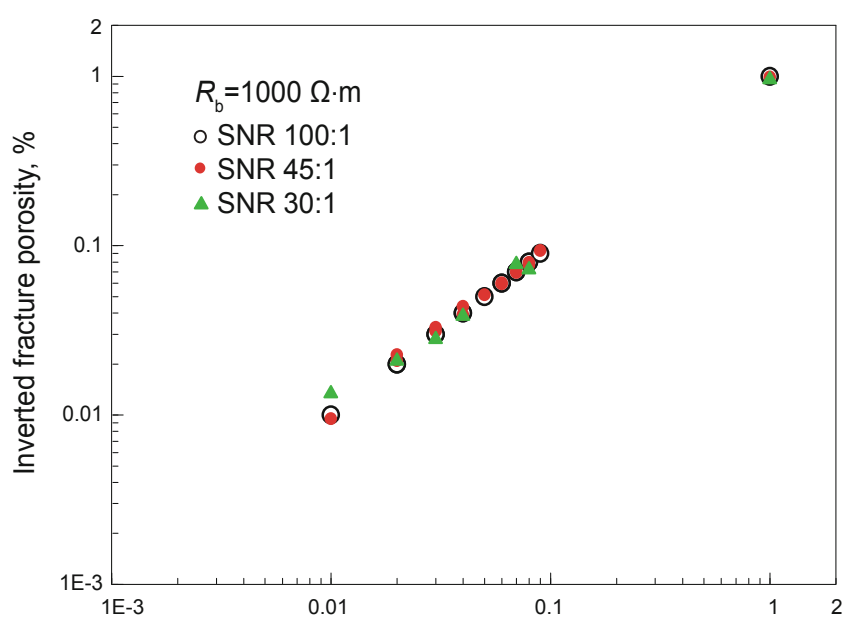

(a) Actual fracture porosity, \%

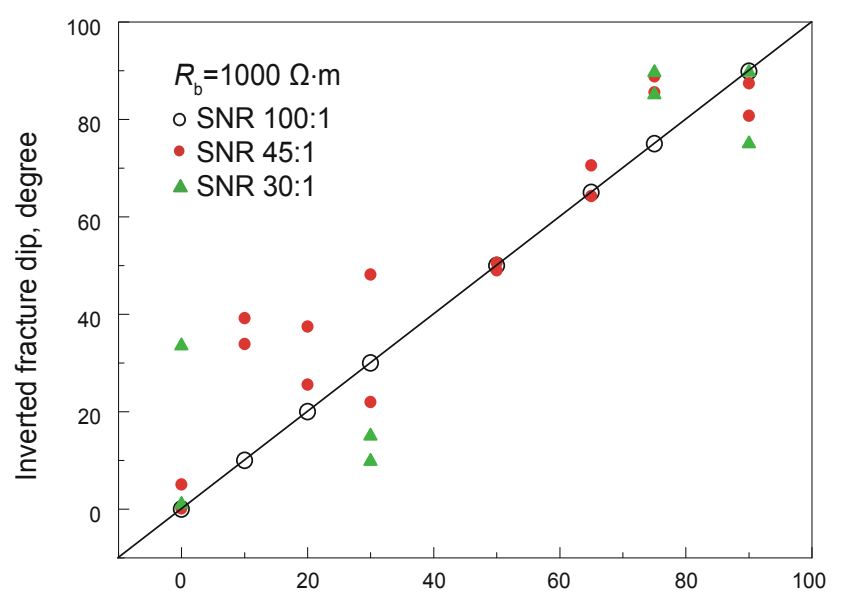

(b) Actual fracture dip, degree

Fig. 8 Inversion results of array lateral logs versus actual formation model

\section{Conclusions}

1) Fracture development makes the formation show electrical anisotropy, which then determines the response of array laterologs in fractured reservoirs. With the fracture dip increasing, the apparent resistivity of an array laterolog becomes greater.

2) The array laterolog response has a good correlation with fracture porosity and dip. With fracture porosity increasing, the response of an array laterolog decreases. The difference between the deep and shallow resistivities of an array laterolog relies on the fracture dip angle. A high angle fractured formation has a positive difference.

3) Because of its sensitivity to fracture, the array laterolog can be used to determine fracture parameters, which is helpful for improving evaluation of fractured formations. Due to limited measuring accuracy in high resistivity formation and the difficulty in determining bulk resistivity, the inversion of the array laterolog can still be semi-quantitative in determining formation parameters.

\section{Acknowledgements}

This research is supported by Shandong Natural Science Foundation (Y2007F25) and Fundamental Research Funds for the Central Universities in China (09CX04001A).

\section{References}

Bang J, Solstad A and Mjaaland S. Formation electrical anisotropy derived from induction log measurements in a horizontal well. SPE Annual Technical Conference and Exhibition, 1-4 October 2000. Dallas, Texas

Chen Y H, Chew W C and Zhang G J. A novel array laterolog method. The Log Analyst. 1998. 39(5): 23-32

Deng S G, Tong Z Q and Fan Y R. Numerical simulation of dual laterolog response in tilted anisotropic formation. Acta Petrolei Sinica. 2006. 27(3): 61-64 (in Chinese)

Keller G V and Frischknecht F C. Electrical Methods in Geophysical Prospecting. Oxford: Pergamon Press. 1996. 123-133

Klein J D and Martin P R. The petrophysics of electrically anisotropic reservoirs. The Log Analyst. 1997. 38(3): 25-36

Li S J, Xiao C W, Wang H M, et al. Mathematical model of dual laterolog response to fracture and quantitative interpretation of fracture porosity. Chinese Journal of Geophysics. 1996. 39(6): 845852 (in Chinese)

Liu Z H and Zhang X. Multi-parameter inversion of array laterolog responses. Journal of Xi'an Shiyou University (Natural Science Edition). 2005. 20(1): 30-33 (in Chinese)

Luo L, Hu P Y and Zhou Z Y. Log identification for fracture in carbonate. Acta Petrolei Sinica. 2001. 22(3): 32-35 (in Chinese)

Philippe A P and Roger N A. In situ measurements of electrical resistivity, formation anisotropy and tectonic context. SPWLA $31^{\text {st }}$ Annual Logging Symposium, 24-27 June 1990. Lafayette, Louisiana

Sibbit A M and Faivre O. The dual laterolog response in fractured rocks. SPWLA $26^{\text {th }}$ Annual Logging Symposium, 17-20 June 1985. Dallas, Texas

Smits J W, Dubourg I, Luling M G, et al. Improved resistivity interpretation utilizing a new array laterolog tool and associated inversion processing. SPE Annual Technical Conference and Exhibition, 27-30 September 1998. New Orleans, Louisiana (SPE 49328)

Wang H N, Yang S D and Chang M C. Fast modeling of lateral resistivity logging in horizontal anisotropic layers and its application. Well Logging Technology. 1998. 22(1): 28-31 (in Chinese)

Wu J, Xie W W, Xie Q C, et al. Forward response analysis of an array lateral logging tool. Journal of Xi'an Shiyou University. 2008. 23(1): 73-76, 80 (in Chinese)

Yang W. Determining resistivity anisotropy of geological formation by joint inversion of lateral and induction logs. Seismology and Geology. 2003. 25(2): 274-278 (in Chinese)

Zhang G J. Electrolog. Beijing: Petroleum Industry Press. 1984. 31-56 (in Chinese)

Zhao J Q, Zhou D, Li X W, et al. Laboratory measurement and applications of anisotropy parameters of rock. SPWLA $35^{\text {th }}$ Annual Logging Symposium, 19-22 June 1994. Tulsa, Oklahoma

(Edited by Hao Jie) 